\title{
Response to letter to the editor by Dr. Vitor Oliveira Carvalho and Guilherme Veiga Guimaraes: Is the 6-min walking test a submaximal exercise test in heart failure patients?
}

\author{
Melissa L. Jehn • Arno Schmidt Trucksäss
}

Accepted: 22 September 2009/Published online: 16 October 2009

(C) Springer-Verlag 2009

We thank Carvalho and Guimaraes (2009) for their interest in our article (Jehn et al. 2009). The primary purpose of our study was to measure the cardio-respiratory response during a constant load exercise test (6-min walk test) in patients with heart failure and compare peak values with an incremental exercise test (ramp protocol). We agree with Carvalho et al. that a treadmill test would have been a more direct comparison to the 6-min walk test regarding exercise mode. However, treadmill tests are rarely done in patients with heart disease in many European countries, in particular Germany. Thus, we wanted our test outcome to be as closely related to its clinical applicability as possible.

In support of our data, it has been previously shown that the cardio-respiratory response and its prognostic utility are not greatly impacted by the mode of exercise in patients with heart failure, and that test outcome of the CPET can be applied globally, irrespective of exercise modality (i.e. treadmill vs. cycle ergometry) (Arena et al. 2005). The importance in eliciting a maximal exercise response lies predominately in the nature of the preferred exercise test, in other words, what the patient feels more comfortable with (Albouaini et al. 2007; Piepoli et al. 2006). This goes for walking versus cycling as well as constant load versus incremental exercise stress test (CPET). The main reason why cycle ergometry often is avoided as means of CPET testing in many western

Communicated by Susan Ward.

M. L. Jehn $(\square)$ · A. S. Trucksäss

Department of Sports Medicine, Institute of Exercise

and Health Sciences, University of Basel, Bierstrasse 320B,

St. Jacobs Turm, 4052 Basel, Switzerland

e-mail: melissa.jehn@unibas.ch countries is usually due to the general inability of individuals in riding a bike, albeit it offers more stability and technically better ECG data which is of particular pertinence in cardiac patients during exercise testing. Within our study population, however, the use of a bicycle acts as a widespread mode of transportation within the general community and most patients felt more comfortable on a cycle ergometer than the treadmill.

Carvalho et al. raise an important aspect by pointing out that the use of prognostic cut-off values for $V_{2}$ peak $(14 \mathrm{ml} / \mathrm{kg}$ per min) provided in the literature (Frankenstein et al. 2007; Parikh et al. 2009) would have added to the clinical importance of our findings. However, considering our small study population size, we wanted to ensure that the groups were as closely matched in size $(N)$ as possible.

Likewise, we do agree that both the use of the Borg scale and the respiratory exchange ratio (RER) are important aspects of ensuring an adequate maximal exercise response during exercise testing, as they offer both subjective and objective measures of exertion, respectively (Carvalho et al. 2009). Both, the Borg scale $(\geq 18)$ and RER $\geq 1.05$, were used as measures of maximal performance in our study, and patients not meeting these criteria were excluded from the analyses.

Our main prerogative was to show that the value in eliciting a maximal exercise response in patients with heart failure lies predominately in choosing the appropriate exercise test and is dependent on the functional status and exercise limitations of that particular patient. Moreover, our findings indicate a constant load exercise test (in which individuals choose their own workload and thus exercise intensity) to elicit higher peak respiratory parameters than incremental stress testing in patients with limited exercise capacity. 


\section{References}

Albouaini K, Egred M, Alahmar A, Wright DJ (2007) Cardiopulmonary exercise testing and its application. Heart 93(10):12851292

Arena R, Guazzi M, Myers J, Ann PM (2005) Prognostic characteristics of cardiopulmonary exercise testing in heart failure: comparing American and European models. Eur J Cardiovasc Prev Rehabil 12(6):562-567

Carvalho VO, Guimaraes GV (2009) Is the 6-min walking test a sub-maximal exercise test in heart failure patients? Eur J Appl Physiol

Carvalho VO, Bocchi EA, Guimaraes GV (2009) The Borg Scale as an important tool of self-monitoring and self-regulation of exercise prescription in heart failure patients during hydrotherapy. Circ J
Frankenstein L, Nelles M, Hallerbach M, Dukic D, Fluegel A, Schellberg D, Katus HA, Remppis A, Zugck C (2007) Prognostic impact of peak $V \mathrm{O} 2$-changes in stable $\mathrm{CHF}$ on chronic betablocker treatment. Int J Cardiol 122(2):125-130

Jehn M, Halle M, Schuster T, Hanssen H, Weis M, Koehler F, SchmidtTrucksass A (2009) The 6-min walk test in heart failure: is it a max or sub-maximum exercise test? Eur J Appl Physiol

Parikh MN, Lund LH, Goda A, Mancini D (2009) Usefulness of peak exercise oxygen consumption and the heart failure survival score to predict survival in patients $>65$ years of age with heart failure. Am J Cardiol 103(7):998-1002

Piepoli MF, Corra U, Agostoni PG, Belardinelli R, Cohen-Solal A, Hambrecht R, Vanhees L (2006) Statement on cardiopulmonary exercise testing in chronic heart failure due to left ventricular dysfunction. Recommendations for performance and interpretation. Eur J Cardiovasc Prev Rehabil 13(1):10-12 